\title{
Efectos del programa educativo "Libres de COVID-19" sobre las habilidades sociales preventivas del adolescente
}

Miryam Lora-Loza*1,aa; Jaimito Sevillano-Saldaña 2,b; Richard García-Ishimine 1,c; Tomas Rodríguez-Beas 3,d; Yuri Vilca-Bejarano 4,e

\section{RESUMEN}

Objetivo: Determinar el efecto del programa educativo Libres de COVID-19 en las habilidades sociales preventivas de adolescentes atendidos en un hospital provincial de Perú.

Materiales y métodos: Investigación explicativa y preexperimental de diseño longitudinal y prospectivo que responde a una preprueba y una posprueba en grupo intacto. La población estuvo conformada por cuarenta adolescentes de ambos sexos, de 13 a 19 años, que fueron atendidos en el Hospital Provincial de Tayabamba. Los criterios de inclusión fueron los siguientes: a) adolescente con edad entre 13 a 19 años, b) tener una historia clínica completa y c) haber sido atendido en el Hospital Provincial de Tayabamba. Las variables estudiadas (antes y después de la aplicación del programa) fueron las habilidades sociales preventivas y sus dimensiones de asertividad, comunicación, autoestima y toma de decisiones. El programa Libres de COVID-19 fue aplicado con una prueba previa y otra posterior. Se empleó un cuestionario de evaluación del desarrollo de habilidades sociales preventivas de cuatro dimensiones y veintisiete reactivos. El análisis no paramétrico de datos longitudinales fue utilizado para una muestra de sujetos evaluados en dos ocasiones en el tiempo.

Resultados: Antes de aplicar el programa educativo Libres de COVID-19, las habilidades sociales preventivas de adolescentes, en general, fueron regulares en el 55,00 \% de los participantes y, después del programa, el $80,00 \%$ obtuvo la calificación de buenas. Al inicio, la asertividad fue regular (45,00\%), y mejoró a buena (75,00 \%) después del programa. La comunicación antes del programa era regular (70,00\%), y después resultó buena (85,00 \% de los adolescentes). La toma de decisiones fue buena en el 50,00\% del grupo antes del programa; y después, el 90,00 \% de los participantes consiguió la misma calificación.

Conclusiones: Las habilidades sociales preventivas de adolescentes atendidos en el Hospital Provincial Tayabamba, así como la asertividad, la comunicación, la autoestima y la toma de decisiones mejoraron significativamente con la aplicación del programa educativo Libres de COVID-19.

Palabras clave: Habilidades sociales; Asertividad; Autoestima; Programa (Fuente: DeCS BIREME).

\section{Effects of the "Free of COVID-19" educational program on teenagers' preventive social skills}

\section{ABSTRACT}

Objective: To determine the effects of the "Free of COVID-19" educational program on the preventive social skills of teenagers treated at a provincial hospital in Peru.

Materials and methods: An explanatory, pre-experimental, longitudinal and prospective research with a pretest and posttest intact group design. The population consisted of 40 male and female teenagers aged between 13 and 19 , who were treated at the Tayabamba provincial hospital. The inclusion criteria were a) teenagers aged between 13 and 19 , b) teenagers with complete medical records and c) teenagers treated at the Tayabamba provincial hospital. The variables (before and after the program) were the preventive social skills and their dimensions: assertiveness, communication, self-esteem and decision-making. The "Free of COVID-19" educational program was implemented with a pretest and a

1. Universidad César Vallejo, Escuela de Posgrado. Trujillo, Perú.

2. Universidad César Vallejo, Hospital Provincial de Tayabamba. La Libertad, Perú.

3. Universidad César Vallejo, Facultad de Ciencias Médicas. Trujillo, Perú.

4. Universidad César Vallejo, Hospital I Luis Albrecht EsSalud. Trujillo, Perú.

a. Obstetra, especialista en Emergencias Obstétricas, Doctora en Gestión y Planificación.

b. Enfermero, Magister en Gestión de los Servicios de la Salud.

c. Químico farmacéutico, Maestría en Gestión del Talento Humano.

d. Ingeniero Mecánico, Magíster en Gestión Ambiental.

e. Médico Cirujano, Magister en Gestión de los Servicios de la Salud.

* Autor corresponsal. 
posttest. A questionnaire made up of four dimensions and 27 questions was administered for assessing the preventive social skills. A non-parametric analysis of longitudinal data was used for a sample of subjects who were evaluated twice during the course of the study.

Results: Before the implementation of the "Free of COVID-19" educational program, the teenagers' preventive social skills were, in general terms, fair (55.00\%) and, after the program, good $(80.00 \%)$. Their assertiveness was fair $(45.00 \%)$ before the program and improved to good $(75.00 \%)$ after the program. Their communication was fair $(70.00 \%)$ before the program and good $(85.00 \%)$ afterwards. Their decision-making was good (50.00 \%) before the program and also good $(90.00 \%)$ after the implementation of the program.

Conclusions: The preventive social skills of teenagers treated at the Tayabamba provincial hospital, as well as their assertiveness, communication, self-esteem and decision-making, significantly improved with the implementation of the "Free of COVID-19" educational program.

Keywords: Social skills; Assertiveness; Self concept; Program (Source: MeSH NLM).

\section{INTRODUCCIÓN}

Uno de los daños colaterales más significativos de la pandemia de la COVID-19 es el deterioro de la calidad de vida del adolescente. Con esta enfermedad llegaron el confinamiento social obligatorio, el cierre de los centros educativos, la prohibición a desarrollar actividades de recreación social, etc., situaciones que generan, necesariamente, mayores niveles de estrés, ansiedad, temor, desconcierto y pánico, nunca vistos en la historia reciente ${ }^{(1)}$. Las familias y los distintos grupos sociales ya no pueden garantizar al adolescente una formación inclusiva y equitativa porque ellos mismos se ven obligados a seguir las políticas y las medidas de contención de la crisis sanitaria mundial. Lógicamente, los adolescentes son las personas menos indicadas para que, a través de sus respuestas e iniciativas personales, puedan ser escuchados, y se desconoce el nivel de vulnerabilidad en que han quedado frente al peligro de enfermar y morir por la enfermedad y/o por los daños que las medidas de contención social le causa a su salud bio-psicosocial (2). La COVID-19 afecta a todo el mundo, pero, según la OPS/OMS, América Latina es una de las regiones que más está sufriendo su impacto, ya que antes de la pandemia, la población adolescente ya estaba en desventaja respecto a Europa o países como China, Rusia y Japón, entre otros. La situación de pobreza y pobreza extrema que contextualizaban la realidad socioeconómica y familiar de los adolescentes alcanzaban entre el $30 \%$ al $45 \%$, en promedio. Con el COVID-19 se ha multiplicado y ha alcanzado una media del $80 \%$. Es decir, la situación de vulnerabilidad de los adolescentes se ha incrementado exponencialmente y se ubican como una población de mayor riesgo o peligro respecto al desarrollo personal y social adecuados ${ }^{(3)}$.

En el Perú, entre el 70 al $90 \%$ de los adolescentes no tiene la mínima oportunidad de proyectarse asertivamente a través de un planeamiento o dominio de sus habilidades sociales; sus formas de comunicarse han sido distorsionadas, eliminando la posibilidad de desarrollar un comportamiento social adecuado a su edad. La autoestima está muy por debajo de los valores normales, lo que genera serios problemas para la salud mental y hasta se han prohibido las prácticas racionales para la tomar decisiones personales, familiares y sociales. El estado actual en que se encuentra la población adolescente vulnera sus derechos y su bienestar futuro (4). En nuestro país, entre el 70 al $80 \%$ de los adolescentes ve afectada su salud mental por la falta de interacción y desarrollo de sus actividades educativas ${ }^{(5)}$. Los programas educativos se definen como un conjunto de planes, estrategias, objetivos y acciones con fines específicos sobre el comportamiento, actitudes y motivaciones de las personas ${ }^{(6)}$. La finalidad de estos planes es variada y diversa; se fundamenta en la trasferencia de información y conocimientos para desarrollar habilidades y destrezas en algún campo específico del conocimiento o comportamiento humano ${ }^{(7)}$.

La estructura de estos programas responde a las conexiones y dinámicas que rigen los procesos de trasformación de comportamientos y/o de las dinámicas del desarrollo de habilidades sociales, intelectuales, culturales, filosóficas, educativas, salubristas, etc. Al ser expresos y claros, su propósito es presentar una visión general de las principales herramientas educativas para la entrega y trasferencia de la información, como los datos estadísticos y las precisiones conceptuales, así como las referencias de experiencias de su aplicación en otras poblaciones ${ }^{(8)}$. Esto contribuye a la claridad y transparencia del papel educador del programa, ya que prevé el cambio y especifica su eficiencia, eficacia, equidad y, en general, su función social en el contexto de la realidad en que se aplica ${ }^{(9)}$. Nuestro trabajo busca mejorar la asertividad, la comunicación, la autoestima y la forma como los adolescentes toman sus propias decisiones para evitar enfermar o morir por COVID-19, o ver deteriorado su bienestar bio-psicosocial, especialmente, respecto a su salud metal, que son los principales elementos de desarrollo de las habilidades sociales preventivas en el adolescente, las mismas que se incorporan, después de un proceso educativo, en términos modelares, en el desarrollo de sus comportamientos futuros ${ }^{(10)}$. 
Estos programas no emplean formas interactivas de negociación para imprimir un carácter de presión y/o generar los cambios en el comportamiento, más bien utilizan los elementos facilitadores de la información y conocimiento para brindar las herramientas de racionalización para el desarrollo de habilidades. Así, las limitaciones para desarrollar nuestras propias iniciativas disminuyen o se eliminan ${ }^{(11)}$.

Desde este punto de vista, las habilidades sociales preventivas se definen como las representaciones formales de las experiencias socioculturales, filosóficas y cognitivas, que resultan de enorme interés al explorar las fuentes de la aplicabilidad de iniciativas para conseguir logros y objetivos de superación de situaciones o comportamientos no deseados. Estas habilidades sociales de prevención no pueden prescindir del apoyo sustantivo de los programas educativos, especialmente cuando la población de interés son los adolescentes. En este caso, bajo las condiciones de confinamiento social y de riesgo de contraer el COVID-19 o de deteriorar su bienestar bio-psicosocial. En otras palabras, no existen fórmulas específicas que determinen las estructuras de los cambios que se persiguen, aunque muchos comportamientos individuales y grupales puedan estar ya dirigidos a aumentar las habilidades sociales preventivas ${ }^{(12)}$. El concepto de prevención que se une al de desarrollo de habilidades sociales nace de la vulnerabilidad de las personas frente a fenómenos sociales, naturales o del pensamiento, como el COVID-19. En consecuencia, las habilidades sociales preventivas de asertividad se definen como procesos que racionalizan las nuevas formas en que las personas (adolescentes) pueden ayudarse a conseguir metas, obtener algún grado de felicitación por su comportamiento, agradecimiento o reconocimiento por guardar la distancia social para evitar el contagio del COVID-19. El punto central es la racionalización de los procedimientos, que tiene como objetivo alcanzar mejores habilidades sociales asertivas rompiendo los extremos negativos que se presentan en las instancias intermedias de la comprensión de la realidad en que se vive. Por ello, el objetivo central de esta investigación fue determinar los efectos del programa educativo Libres de COVID-19 en las habilidades sociales preventivas de los adolescentes atendidos en un hospital provincial del Perú en 2020.

\section{MATERIALES Y MÉTODOS}

\section{Diseño y población de estudio}

La investigación es de tipo explicativa (en su nivel de preexperimental) y de diseño longitudinal. Responde a una preprueba y una posprueba en grupo intacto no probabilístico por asignación o interés. La población objetivo estuvo conformada por 40 adolescentes de ambos sexos, entre 13 a 19 años, que fueron atendidos en el Programa de Atención Integral del Escolar y Adolescente en el Consultorio Externo del Hospital Provincial de Tayabamba, región La Libertad, entre los meses de junio a diciembre 2020. Los criterios de inclusión fueron los siguientes: a) adolescentes de ambos sexos entre 13 a 19 años que acepten participar voluntariamente en el estudio y b) adolescentes con historias clínicas completas.

\section{Variables y mediciones}

Las variables estudiadas (antes y después del programa) fueron las habilidades sociales preventivas $y$ sus dimensiones: de asertividad, de comunicación, de autoestima y de toma de decisiones. Se empleó un cuestionario de evaluación del desarrollo de habilidades sociales preventivas en adolescentes con la prueba de ingreso y salida inherentes al programa educativo "Libres de COVID-19", que aborda las 4 dimensiones del desarrollo de habilidades sociales preventivas antes mencionadas, con 27 reactivos. Luego se aplicó el programa educativo con pretest de ingreso y postest. Como recursos de comunicación se utilizaron la plataforma Zoom y las aplicaciones E-learning propuestas por el Ministerio de Salud (Minsa 2005), adaptadas en función del desarrollo de habilidades sociales preventivas contra el COVID-19. Las sesiones tenían una duración de 40 minutos y la consultoría en línea estaba abierta en cualquier momento.

\section{Análisis estadístico}

Para estimar el efecto de la aplicación del programa educativo con un pretest de ingreso y un postest, se emplearon las metodologías descritas por Brunner et al. y sus aplicaciones prácticas en el análisis de datos longitudinales de experimentos que estudian estudian una sola muestra de sujetos en dos ocasiones en el tiempo (pre y postest) ${ }^{(13)}$. En este caso, el experimento consiste en evaluar la respuesta de las unidades de investigación sometidas a una condición en particular (programa educativo) en dos ocasiones (pretest y postest; $t=2$ ); el mismo se constituye en el diseño experimental más simple en el contexto de los datos longitudinales. Aquí, el modelo estadístico para $t=2$ períodos considera que los vectores aleatorios observados $X_{-k}=\left(X_{-k 1}, X_{-k 2}\right)^{\prime}, k=1, \ldots, n$, son independientes, $y$ las $X_{-k s}$ tienen la función de distribución marginal $F_{-s, s}=1,2$.

En la tabla 1 se presentan los datos y las distribuciones para esta clase de diseño de experimentos. 
Tabla 1. Observaciones y funciones de distribución para un diseño donde se estudia un solo grupo de sujetos evaluados en dos períodos de tiempo $t=2$

\begin{tabular}{cccccc}
\hline & \multicolumn{2}{c}{ Vatos } & Distribuciones marginales \\
& \multicolumn{2}{c}{ Tiempo } & \multicolumn{2}{c}{ Tiempo } \\
\hline Unidad de investigación & $\mathrm{s}=1$ & $\mathrm{~s}=2$ & $\mathrm{~s}=1$ & $\mathrm{~s}=2$ \\
$\mathrm{k}=1$ & $\mathrm{X}$ 11 & X_12 & F_1 & F_2 \\
$\vdots$ & $\vdots$ & $\vdots$ & $\vdots$ & $\vdots$ \\
k=n & X_n1 & X_n2 & F_1 & F_2
\end{tabular}

De esta manera, la hipótesis puede ser formulada como $H_{0}^{F}: F_{1}=F_{2}$, esto sugiere que las dos funciones de distribución marginal $F_{1}$ y $F_{2}$. Asimismo, la hipótesis $H_{0}^{p}: p=\int F_{1} d F_{2}=\frac{1}{2}$ significa que una diferencia entre las distribuciones $F_{1}$ y $F_{2}$ se acepta siempre que la tendencia central no cambie, es decir, los efectos relativos de tratamiento $p_{s}=\int H d F_{s}, s=1,2$ son iguales $\mathrm{y}$, en consecuencia, $p_{2}-p_{1}=p-\frac{1}{2}=0$. En ese caso, $H=\frac{1}{2}\left(F_{1}+F_{2}\right)$. La hipótesis $H_{0}^{F}$ es más robusta que $H_{0}^{p}$ debido a que $F_{1}=F_{2}$ implica que $p_{2}=p_{1}$.

Para calcular los estadísticos y probar la hipótesis $H_{0}^{F}$, solo se necesitan los rangos de las $N=2 n$ observaciones, mientras que para probar la hipótesis $H_{0}^{p}: p=\frac{1}{2}$, o su equivalente $p_{2}=p_{1}$, serán necesarios otros rangos. De esta manera, se tiene que $S_{n, 0}^{2}=\frac{1}{n-1} \sum_{k=1}^{n}\left(R_{k 2}-R_{k 1}\right.$ $\left.\bar{R}_{.2}+\bar{R}_{.1}\right)^{2}$ representa la varianza empírica de las diferencias de rango $R_{k 2}-R_{k 1}, k=1, \ldots, n$. Así, para muestras grandes, el estadístico $T_{n}^{F}=\sqrt{n} \frac{\bar{R}_{.2}+\bar{R}_{11}}{S_{n, 0}}$ tiene una distribución normal estándar $N(0,1)$ bajo la hipótesis $H_{0}^{F}$. Por el contrario, para muestras pequeñas, la distribución del estadístico $T_{n}^{F}$ se aproxima a una distribución $t$ de Student centrada con $n-1$ grados de libertad. En ese sentido, Brunner et al. refieren que estudios de simulación han demostrado que esta aproximación es bastante buena para tamaños de muestra $n \geq 7$ y distribuciones continuas (13). Para las distribuciones discretas, la calidad de la aproximación depende del número de empates en la muestra: así, para tamaños de muestra $n \geq 15$, esta aproximación es satisfactoria si no hay muchos empates.

La hipótesis $H_{0}^{p}: p=\int F_{1} d F_{2}=\frac{1}{2}$ contiene todas las distribuciones marginales simétricas $F_{1}^{S}$ y $F_{2}^{S}$ con el mismo centro de simetría $\mu_{1}^{s}=\mu_{2}^{s}$ pero diferentes varianzas $\sigma_{1}^{S}$ y $\sigma_{1}^{S}$. En ese sentido, esta configuración es conocida como el caso no paramétrico de BehrensFisher. Así, se pueden estimar los efectos relativos de tratamiento $p_{1}$ y $p_{2}$ de la misma manera como en el caso de la hipótesis $H_{0}^{F}: F_{1}=F_{2}$. En ese orden, la varianza del estadístico $U_{n}=\sqrt{n}\left(\hat{p}-\frac{1}{2}\right)$ bajo la hipótesis $H_{0}^{p}$ es distinta a la estimada bajo la hipótesis $H_{0}^{F}$, por lo que bajo la hipótesis $H_{0}^{p}$, un estimador consistente para la varianza del estadístico $U_{n}$ es dado mediante $S_{n, p}^{2} / n^{2}$, donde

$S_{n, p}^{2}=\frac{1}{n-1} \sum_{k=1}^{n}\left[\left(R_{k 2}-R_{k 2}^{(2)}\right)-\left(R_{k 1}-R_{k 1}^{(1)}\right)-\left(\bar{R}_{.2}-\bar{R}_{.1}\right)^{2}\right]$

\section{Consideraciones éticas}

El trabajo respeta los valores éticos y morales inherentes a la aplicación de investigaciones experimentales en línea (en el campo virtual) y también los derechos legales de los adolescentes, de acuerdo con pautas éticas internacionales para la investigación relacionadas con la salud de seres humanos elaboradas por el Consejo de Organizaciones de las Ciencias Médicas (CIOMS) en colaboración con la Organización Mundial de la Salud (OMS) y la Organización Panamericana de la Salud (OPS) (2016) y del informe Belmont sobre el respeto a las personas, beneficencia y resguardo su independencia. Asimismo, se respetaron los procedimientos de "no copia" verificando la originalidad del estudio con el programa Turnitin, como establece la Escuela de Posgrado de la Universidad César Vallejo.

\section{RESULTADOS}

Antes de aplicar el programa "Libres de COVID-19", las habilidades sociales preventivas de los adolescentes atendidos en el Hospital Provincial Tayabamba fueron malas (en el 10,00\% de los participantes), regulares (en el $55,00 \%$ ) y buenas (en $35,00 \%$ ); y después del programa habían cambiado a malas $(0,00 \%)$, regulares $(20,00 \%)$ y buenas $(80,00 \%)$. Asimismo, en relación a las dimensiones de las habilidades sociales preventivas, encontramos que los valores de la asertividad, previos al programa, fueron malos (15,00\% de los adolescentes), regulares (en $45,00 \%$ del grupo) y buenos (en el $40,00 \%$ ); después del programa fueron malos $(5,00 \%)$, regulares $(20,00 \%)$ y buenos $(75,00 \%)$. Al inicio del estudio, las habilidades de comunicación en el 15,00\% de los participantes fueron malas; regulares, en el $70,00 \%$; y 
buenas, en el 15,00 \%; después del programa fueron malas $(5,00 \%)$, regulares $(10,00 \%)$ y buenas $(85,00 \%)$.

Antes de aplicar el plan "Libres de COVID-19", la autoestima, era mala en el 5,00 \% de los adolescentes; regular, en el $65,00 \%$; y buena en $30,00 \%$ del grupo; y después del programa encontramos el mismo porcentaje con calificación mala, el grupo de nivel regular bajó a 10,00 \% y el grupo de calificación buena aumentó al 85,00\%. La toma de decisiones de los adolescentes atendidos en el Hospital Provincial Tayabamba antes del programa educativo Libres de COVID-19 eran malas (15,00\%), regulares $(35,00 \%)$ y buenas (50,00\%); y después del programa obtuvimos lo siguiente: malas $(5,00 \%)$, regulares $(5,00 \%)$ y buenas $(90,00 \%)$.

Tabla 2. Efectos del programa educativo "Libres de COVID-19", sobre las habilidades sociales preventivas y sus dimensiones del adolescente atendido en el Hospital Provincial Tayabamba, 2020

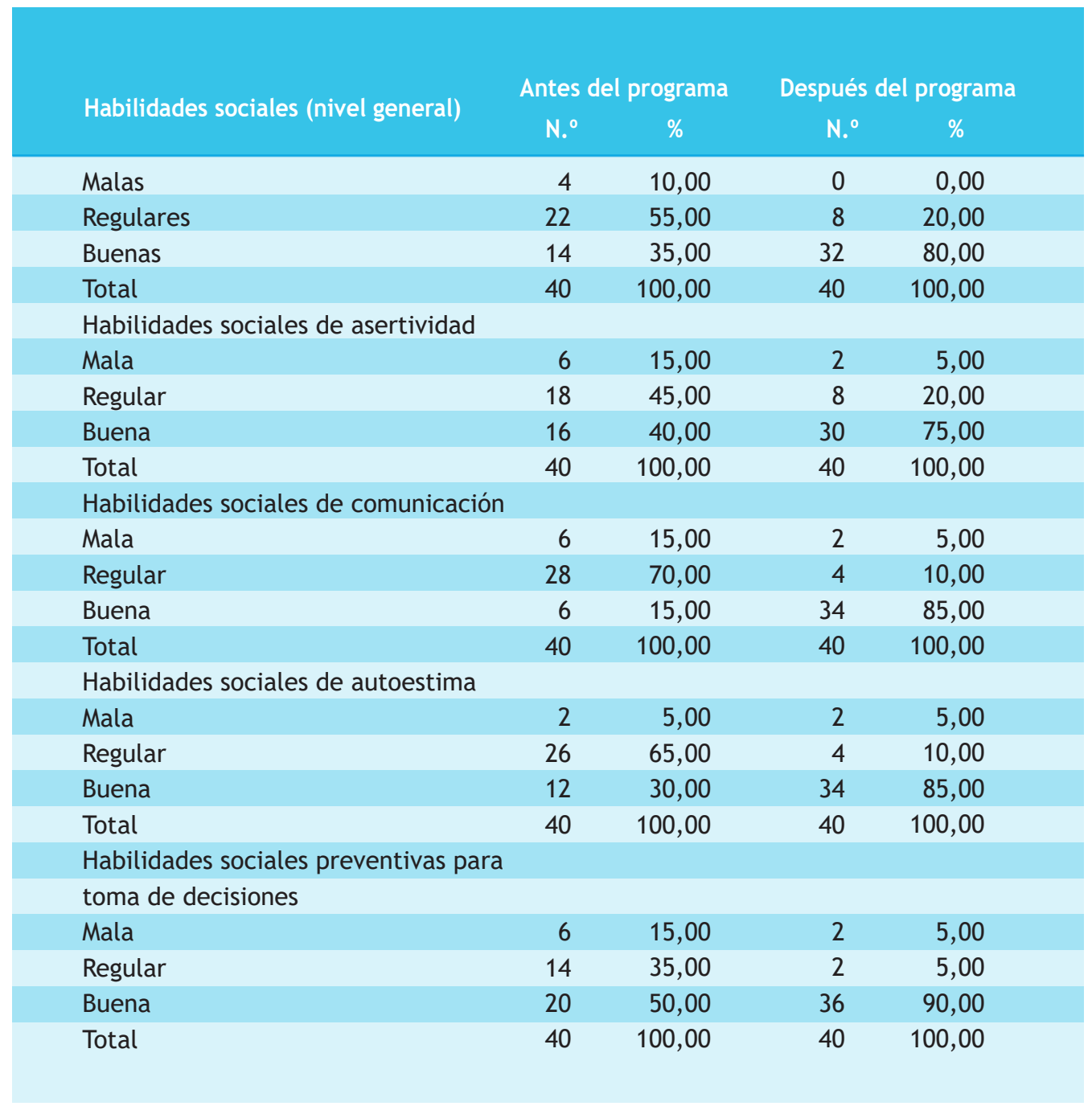

Las estimaciones puntuales de los efectos relativos del programa educativo "Libres de COVID-19" sobre las habilidades sociales preventivas y sus dimensiones del adolescente atendido en el Hospital Provincial Tayabamba en 2020 se incrementan después de que se aplica el programa educativo (Figuras 1 y 2 ). 


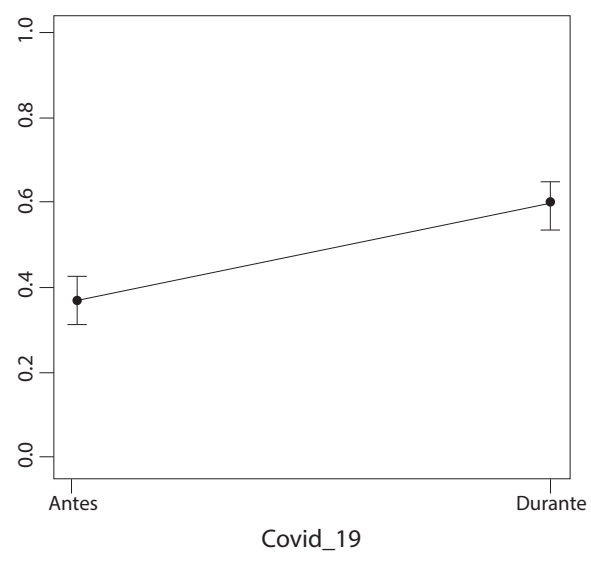

Figura 1. Intervalos de confianza de $95 \%$ para los efectos relativos del programa educativo "Libres de COVID-19" sobre las habilidades sociales preventivas del adolescente atendido en el Hospital Provincial Tayabamba, 2020

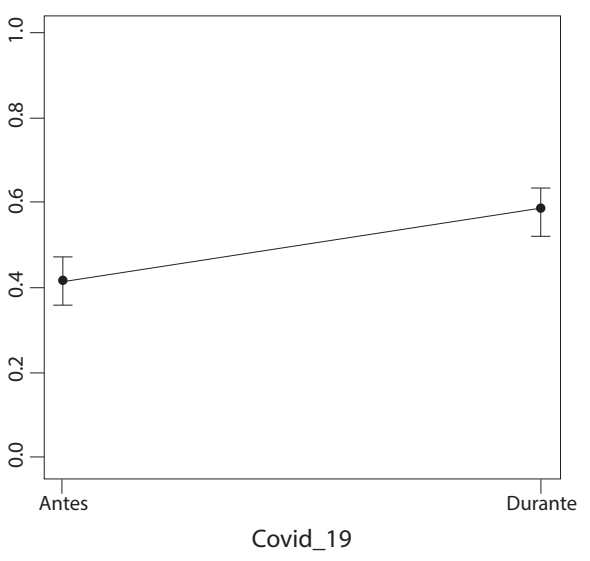

Asertividad

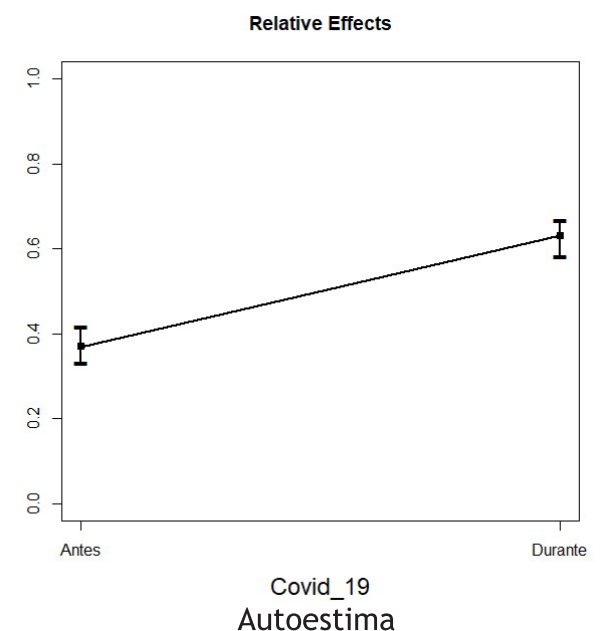

Relative Effects

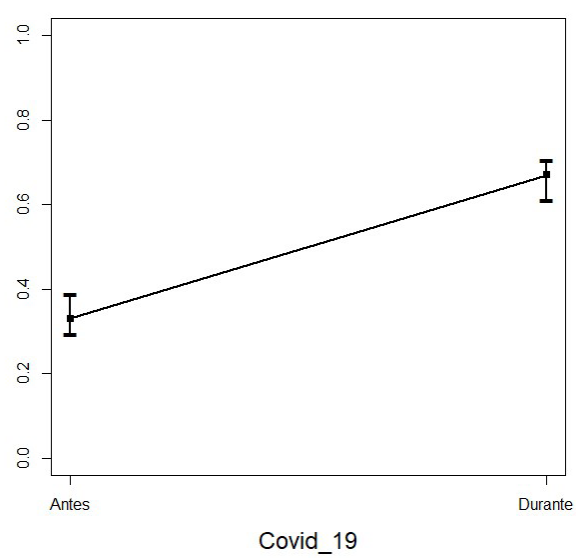

Comunicación

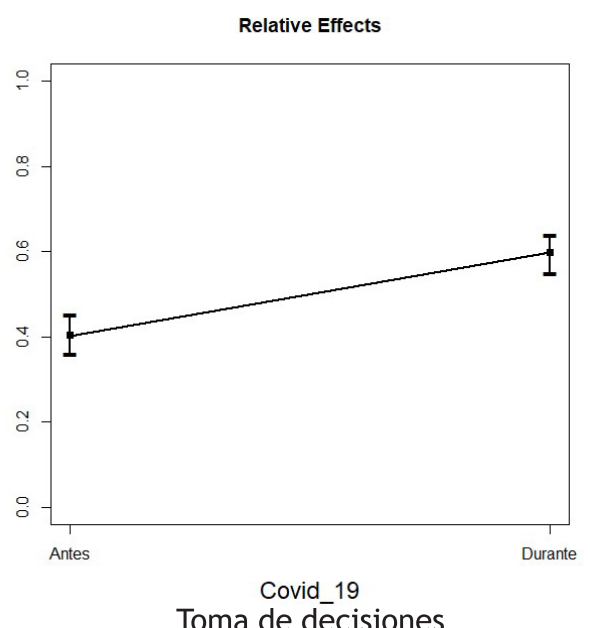

Figura 2. Intervalos de confianza de $95 \%$ para los efectos relativos del programa educativo "Libres de COVID-19" sobre las dimensiones asociadas a las habilidades sociales preventivas del adolescente atendido en el Hospital Provincial Tayabamba, 2020 
Efectos del programa educativo "Libres de COVID-19" sobre las

habilidades sociales preventivas del adolescente

El análisis no paramétrico sugiere que el comportamiento de las habilidades sociales preventivas y sus dimensiones del adolescente variaron significativamente $(p<0,05)$ después de que se aplicó el programa educativo "Libres de COVID-19" (Tabla 3).

Tabla 3. Efectos del programa educativo "Libres de COVID-19" sobre las habilidades sociales preventivas y sus dimensiones del adolescente atendido en el Hospital Provincial Tayabamba, 2020

\begin{tabular}{ccccc}
\hline Variable & \multicolumn{2}{c}{ Datos } & \multicolumn{2}{c}{ Distribuciones marginales } \\
\hline & \multicolumn{2}{c}{ Tiempo } & \multicolumn{2}{c}{ Tiempo } \\
\hline Unidad de investigación & $\mathrm{s}=1$ & $\mathrm{~s}=2$ & $\mathrm{~s}=1$ & $\mathrm{~s}=2$ \\
$\mathrm{k}=1$ & $\mathrm{X}-11$ & $\mathrm{X}-12$ & $\mathrm{~F}-1$ & $\mathrm{~F}-2$ \\
\hline$\vdots$ & $\vdots$ & $\vdots$ & $\vdots$ & $\vdots$ \\
$\mathrm{k}=\mathrm{n}$ & X_n1 & X_n2 & F_1 & F_2 \\
\hline
\end{tabular}

\section{DISCUSIÓN}

Los resultados muestran que el adolescente atendido en el Hospital Provincial Tayabamba desarrolló mejoró sus habilidades sociales preventivas con la ayuda del programa educativo "Libres de COVID-19". Antes del estudio ya existía un desarrollo básico de las habilidades sociales preventivas, las mismas que fueron repotenciadas, tanto por la labor preventiva de enfermería, como por una actitud de respuesta a los temores, incertidumbres, miedos y pánicos que pudo y puede generar el COVID-19 (14). Las labores de la enfermería respecto a la atención primaria de la salud (que están orientadas a cambiar comportamientos, mejorar conocimientos y desarrollar habilidades) pudieron tener un éxito relativo al aplicar el programa; sin embargo, la idea primaria del proyecto fue que el adolescente mismo pueda generar sus propios procesos de autocuidado para evitar enfermar de COVID-19, pero las necesidades relativas para desarrollar habilidades sociales preventivas fueron mayores y marcaron el camino para romper sus formas de suprimir casi todos los tipos de conductas o comportamientos preventivos de interconexión con la realidad que vive ${ }^{(15)}$. Los resultados también indican que la asertividad, la comunicación, la autoestima y la toma de decisiones fueron los elementos primarios para mover los indicadores de desarrollo de estas habilidades. Por ello, podemos señalar que estamos frente a una nueva descripción del papel de la enfermería en el área de la atención primaria de la salud en la población adolescente ${ }^{(12)}$.

En ese sentido, nuestros resultados muestran la importancia social de los aprendizajes adaptativos a las nuevas formas de convivencias o a las nuevas formas de establecer las relaciones sociales para el cuidado de su salud ${ }^{(16)}$.

Las implicancias de esta pandemia, como el confinamiento obligatorio o el distanciamiento social, cambiaron las formas de interacción comunitaria.

En consecuencia, las conductas y motivaciones para el desarrollo de nuevas habilidades sociales preventivas se trastornan.

A partir de los resultados, planteamos que la enfermería cambia su rol asistencial a un papel facilitador de los procesos integradores de los comportamientos médicos y sociales preventivos, basados en los postulados del aprendizaje social ${ }^{(17,18)}$ y las teorías del autocuidado de Orem (19), ambos implicados en aspectos puntuales de los supuestos basales de la teoría de la jerarquía de las necesidades ${ }^{(20)}$. La información sobre salud pública requiere ser más específica. Hoy, esta información se dirige, específicamente, a los adolescentes, en quienes el carácter agresivo e indescifrable de la COVID-19 (por su variabilidad y diversidad en la presentación y expansión) hace que se enfaticen los mensajes destinados para protegerlos, ya que se alinean con los valores morales, apelan al consenso social o normas científicas, resaltan la perspectiva de aprobación de grupos sociales amplios y tienden a ser persuasivas ${ }^{(21)}$.

Desde el campo de la enfermería, los procesos de aprendizaje para el desarrollo de habilidades sociales preventivas se circunscriben a la proyección del trabajo médico-social hospitalario. Si esta proyección ayuda al adolescente a interactuar mejor con su realidad, entonces se puede decir que un programa educativo, como "Libres de COVID-19", se trasforma en una herramienta capaz de satisfacer las necesidades del cuidado de la salud a nivel preventivo promocional, ya que parte de un acto consiente del adolescente y proporciona al adolescente comodidad y apoyo. El profesional de enfermería se preocupa del bienestar físico y de la salud mental y social; además, asume el rol de mejorar los comportamientos frente a la realidad en que vive el adolescente con el COVID-19. Desde este punto de vista, todas las actividades del programa educativo "Libres de COVID-19" han cumplido con su objetivo de ayudar al adolescente a buscar nuevas formas de interdependencia social en medio de la coyuntura de la pandemia ${ }^{(22)}$. Con base en los resultados, podemos 
afirmar que no solo los adolescentes precisan este tipo de programas. Toda persona debe estar preparada para enfrentar la desinformación con datos precisos y contraargumentos Esta preparación debe concluir antes de que encuentren teorías de conspiración, noticias falsas u otras formas de información errónea. El estudio ha demostrado que se puede "vacunar" a las personas contra las informaciones falsas ${ }^{(14)}$.

Desde esta perspectiva, los programas como "Libres de COVID-19" se presentan como una herramienta que desacelera la trasmisión viral y provoca cambios significativos en los comportamientos. Sin embargo, es necesario reconocer que los aspectos sociales y culturales influyen en el alcance y la velocidad del cambio de comportamiento. Por ello, se deben adoptar y aplicar nuevas y creativas formas de trabajo, sobre todo aquellas que parten de la descripción de contextos sociales específicos, del reconocimiento de las normas y que impidan la desigualdad social, cultural o la polarización. Estos elementos pueden ayudar a tomar mejores decisiones en el momento de identificar los factores de riesgo de comportamientos negativos frente al COVID-19 e intervenir de manera efectiva (2,3). En conclusión, las habilidades sociales preventivas de adolescentes atendidos en el Hospital Provincial Tayabamba, así como las habilidades sociales preventivas de asertividad, comunicación, autoestima y toma de decisiones mejoran significativamente con la aplicación del programa educativo "Libres de COVID-19", ya que antes del programa estas habilidades estaban en un nivel regular y, después de este, mejoraron a un nivel bueno.

Contribuciones de los autores: Todos los autores participaron en la generación y diseño del estudio, recolección, análisis e interpretación de los datos, redacción del manuscrito, revisión crítica del manuscrito y aprobación de la versión final.

Fuentes de financiamiento: Este artículo ha sido financiado por los autores.

Conflicto de intereses: Los autores declaran no tener ningún conflicto de intereses.

\section{REFERENCIAS BIBLIOGRÁFICAS}

1. World Health Organization. Overview of public health and social measures in the context of COVID-19: Interim guidance [Internet]. OMS; 2020. Disponible en: https://apps.who.int/iris/ handle/10665/332115

2. Anderson RM, Heesterbeek H, Klinkenberg D, Déirdre Hollingsworth T. How will country-based mitigation measures influence the course of the COVID-19 epidemic?. Lancet. 2020; 395(10228): 931-4.

3. UNICEF. Impacto de la crisis por COVID-19 sobre los niños y niñas más vulnerables. Reimaginar la reconstrucción en clave de derechos de infancia [Internet]. UNICEF; 2020. Disponible en: https://www. observatoriodelainfancia.es/ficherosoia/documentos/7228_d_ covid19-infancia-vulnerable-unicef.pdf

4. Alto Comisionado Contra la Pobreza Infantil. Brecha Digital y Pobreza Infantil. Documento Breve 14 [Internet]. ACCPI: España; 2020. Disponible en: https://www.comisionadopobrezainfantil.gob.es/ sites/default/files/Brecha\%20digital_DB.pdf

5. Stiff $C$. The game theory of panic-buring- and how to reduce it [Internet]. The Conversation: United States; 2020. Disponible en: http://theconversation.com/the-game-theory-of-panic-buying-andhow-to-reduce-it-134107

6. Ibarra MS. Definición de programa educativo. España: Actualizado. 2015; 22: 34

7. Brooks SK, Webster RK, Smith LE, Woodland L, Wessely S, Greenberg $\mathrm{N}$, et al. El impacto psicológico de la cuarentena y cómo reducirlo: revisión rápida de la evidencia. Lancet. 2020; 395(10227): 912-20.

8. Bailey J. Closing Schools To Slow a Pandemic [Internet]. Education Next: Canada; 2020. Disponible en: https://www.educationnext. org/closing-schools-to-slow-a-pandemic-coronavirus-covid-19public-health/

9. Mällinen S. Teacher effectiveness and online learning. En: Teaching \& Learning. Londrés: Routledge; 2018. p. 139-49.

10. Harris D. Using federal stimulus to get schools through the coronavirus crisis: The case for summer school and summer teacher pay [Internet]. Brookins Institution; 2020. Disponible en: https:// www. brookings.edu/blog/brown-center-chalkboard/2020/03/11/ using-federal-stimulus-to-getschools-through-the-coronavirus-crisisthe-case-for-summer-school-and-summer-teacher-pay/

11. Loeb S. How covid-19 is interrupting children's education [Internet]. The Economist: London; 2020. Disponible en: https:// www.economist.com/international/2020/03/19/how-covid-19-isinterruptingchildrens-education.

12. Zhang J, Litvinova M, Liang Y, Wang Y, Wang W, Zhao S, et al. Changes in contact patterns shape the dynamics of the COVID-19 outbreak in China. Science. 2020; 368(6498): 1481-6.

13. Brunner E, Puri ML. Nonparametric methods in factorial designs. Statistical Papers. 2001; 42: 1-52.

14. UNICEF. Salud mental e infancia en el escenario de la COVID-19: Propuestas de Unicef España [Internet]. UNICEF; 2020. Disponible en: https://www.unicef.es/publicacion/salud-mentale-infancia-enel-escenario-de-la-covid-19-propuestas-de-unicef-espana

15. Organización Mundial de la Salud, Organización Panamericana de la Salud. Principales orientaciones de la comunicación de riesgos [Internet]. OPS-OMS; 2020. Disponible en: www.Downloads/guia\%20 lideres-\%20comunicacion-de-\%20riesgo\%20COVID19.pdf

16. Skinner BF. Teoría del Comportamiento Humano. España: Ariel; 1938.

17. Vigotsky L. Teoría de Aprendizaje y Desarrollo. España: Ariel; 1932.

18. Orem, D. Nursing: concept of practice. 6a ed. New York: Elsevier Mosby; 2001.

19. Maslow A. Teoria de la jerarquía de necesidades. México: Mc Graw Hill; 1943.

20. Lumn P, Cameron B, Ciarán L, McGowan F, Timmons S, Robertson D. Usar la ciencia del comportamiento para ayudar a combatir el coronavirus. Documento de trabajo de ESRI N 656 [Internet]. 2020. Disponible en: http://aei.pitt.edu/102644/

21. Wise T, Zbozinek TD, Michelini G, Hagan CC, Mobbs D. Changes in risk perception and protective behavior during the first week of the COVID-19 pandemic in the United States. R Soc Open Sci. 2020.

22. Lees J, Cikara M. Las metapercepciones de grupo inexactas impulsan atribuciones negativas fuera del grupo en contextos competitivos. Nat Tararear Behav. 2020; 4: 279-86. 
Efectos del programa educativo "Libres de COVID-19" sobre las habilidades sociales preventivas del adolescente

Correspondencia:

Miryam Lora Loza

Dirección: Los Laureles 474 Urb. California. Distrito Trujillo.

La Libertad, Perú.

Correo: mlora@ucv.edu.pe

Teléfono: +51964 257604

Recibido: 15 de marzo de 2021

Evaluado: 09 de abril de 2021

Aprobado: 25 de mayo de 2021

( $)$ La revista. Publicado por Universidad de San Martín de Porres, Perú. (c) Br Licencia de Creative Commons Artículo en acceso abierto bajo términos de Licencia Creative Commons Atribución 4.0 Internacional. (http://creativecommons.org/licenses/by/4.0/)

ORCID iDs

Jaimito Sevillano-Saldaña 1 https://orcid.org/0000-0003-3500-4151

Miryam Lora-Loza C https:// orcid.org/0000-0001-5099-1314

Richard García-Ishimine (ㄱ) https://orcid.org/0000-0002-6675-9779

Tomas Rodríguez-Beas (i) https://orcid.org/0000-0001-8104-3544

Yuri Vilca-Bejarano ittps://orcid.org/0000-0003-3764-3993 